\title{
A simulation method to estimate closing forces in car-sealing rubber elements
}

\author{
J. Ordieres-Meré
}

V. Muñoz-Munilla

A. Bello-García

\section{A. González-Marcos}

\begin{abstract}
The door-closing process can reinforce the impression of a solid, rock-proof, car body or of a rather cheap, flimsy vehicle. As there are no real prototypes during rubber profile bidding-out stages, engineers need to carry out non-linear numerical simulations that involve complex phenomena as well as static and dynamic loads for several profile candidates. This paper presents a structured virtual design tool based on FEM, including constitutive laws and incompressibility constraints allowing to predict more realistically the final closing forces and even to estimate sealing overpressure as an additional guarantee of noise insulation. Comparisons with results of physical tests are performed.
\end{abstract}

Keywords: numerical simulation; product design; large deformations; viscoelastic rubber; nonlinear contact problem; viscoelastic properties; sealing and mechanical operations for rubber. 
Reference to this paper should be made as follows: Ordieres-Meré, J., Muñoz-Munilla, V., Bello-García, A. and González-Marcos, A. (2012) 'A simulation method to estimate closing forces in car-sealing rubber elements', Int. J. Vehicle design, Vol. 59, No. 4, pp.249-271.

Biographical notes: Joaquín Ordieres-Meré is a full Professor at Universidad Politécnica de Madrid. He has research interests closely related to knowledge management and knowledge discovering, both from data and from models, including FEM techniques. He received his $\mathrm{PhD}$ from UNED (Spain) and he was visiting professor at INRIA (France), University of Iowa (EEUU) and ESTI (France). His experience includes processes like steel production (flat and section products), the extrusion of rubber for automotive components and environmental applications for waste management plants.

Víctor Javier Muñoz-Munilla received the MSc Degree of Inductrial Engineer in 1999 from the University of La Rioja, Spain. From 2000 to 2011 he works at an Automotive Sealing systems company and ocassionally teaches at the University of La Rioja, as an Associate Professor. His main research interests are aimed at the FEA programming tools (related to rubber industry), and the application of Artificial Vision for Industrial Processes.

Antonio Bello-García is a full Professor of Computer Graphics in Engineering at the University of Oviedo, Spain. His research is focused on Computer Aided Design (CAD), computer graphics and data mining and visualisation. $\mathrm{He}$ teaches courses on CAD/CAM, optimisation techniques in engineering and digital image processing. He has participated in more than 30 national and international projects, funded by the European Commission and the Spanish Ministry for Science and Innovation.

Ana González-Marcos received the MSc Degree of Industrial Engineer in 2001 and the $\mathrm{PhD}$ Degree in 2006, both from the University of La Rioja, Spain. From 2003 to 2007 she taught at the University of León, Spain. In 2007 she moved to the University of La Rioja, where she is an Associate Professor of Project Management in the Department of Mechanical Engineering. Her research interests include the application of multivariate analysis and artificial intelligence techniques to improve the quality of industrial processes. Her research projects usually concern real processes, such as those in steel making and the automotive industries.

\section{Introduction}

There are increasing requirements for all aspects of cars. Although the sound of a car door closing is unrelated objectively to the intrinsic qualities of the vehicle, it is an important subjective parameter for vehicle evaluation. The closing sound can reinforce the impression of a solid, rock-proof car body or of a rather cheap, flimsy vehicle. To ensure that their vehicles project a quality image, carmakers are issuing more demanding specifications for sealing systems, and specific actions must be adopted during the early phases of design to take care of them (Wagner et al., 1997).

In addition to questions about the insulating properties of rubber that are related to the ability to appropriately fill the gap between the car door and the car itself, there is a limit to the maximum force that should be permitted when closing the door. This is necessary 
as a higher filling capability requires a higher closing force, which could become quite inconvenient for users, if it is not restricted. Thus, it is important to assess the necessary door-closing force at an early stage during the design of the rubber profile and in connection with the rubber's insulation properties (Piateck, 1987).

As there are no real prototypes during the bidding-out periods of computerised designs (CAE), engineers need to carry out highly non-linear, numerical simulations of events that involve complex mechanical phenomena. These include fatigue (Pan and Chai, 2009) and thermal effects and vibrations (Karen et al., 2010), as well as static and dynamic loads for several profile candidates. This enables engineers to take all effects into account when making competitive choices. These simulations also extend to the design of tooling elements (Ordieres-Meré et al., 2003).

The contact problem of a rubber notch with a rigid wedge is analysed in Stenti et al. (2006), based on the large strain theory. The behaviour of rubber is classified as hyper-elastic, in which the strain energy density function can be defined. The choice of reference configuration influences the kinematics, kinetics and constitutive law of the material. As elastomers are viscoelastic materials, their mechanical properties are strain-, frequency - and temperature-dependent.

The design process begins with CAD models, which the design department creates, and is subject to a three-part process that includes finite-element modelling, multi-body dynamic simulations and tolerances (Nash et al., 2009). These must be done accurately and in the least possible time. These simulations are significant, as they enable the production of valid sections, even during preproduction stages, and the minimisation of additional costs for section rebuilding and line reconfiguration (see Figure 1). Figure 1 shows the complexity of the tooling system around the die extrusion head. In addition, more than $150 \mathrm{~m}$ of other equipment are involved. This shows how expensive it is to conduct trials and, because of this, how interesting simulation approaches are.

Figure 1 Extrusion die configuration for production of Ethylene Propylene Diene Monomers (EPDM) (see online version for colours)

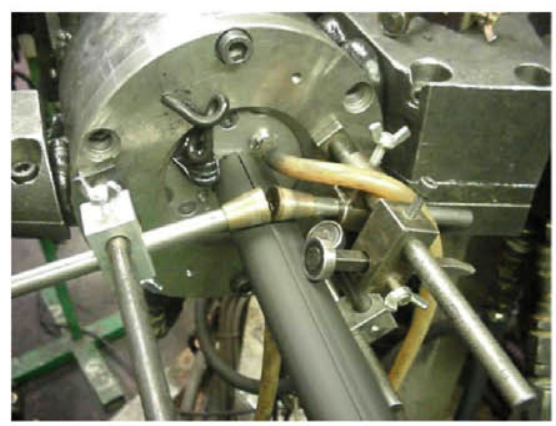

In the design of sealing systems, it is necessary to ensure that there is a pressure seal between the bodywork of vehicles and the profiles of the elastomers.

However, several factors increase the complexity of these designs: The paths or surfaces where anchors should obtain closed joints are composed of complex curves with variations in concavity/convexity and radii of curvature, warping angles, thickness and discontinuities of sheet metal, etc. Thus, the design of the seal must provide geometric stability of the profile when adapting to subject areas. For instance, it is important to achieve an adequate deformation of the profile with the contact surface to avoid defects 
in the form of wrinkles or deformations. Furthermore, the seal's design must provide continuity of the joint along the entire line of the sealing closure.

The distances or gaps to be sealed vary, depending on the specifications of different manufacturers. However, it is a common practice to design nominal tolerances to close the gap surfaces. Also, it is usual to design a blow-up to ensure a continuous contact that causes the interior of the car to be soundproof. This blow-up creates a suction effect of the air on the mobile areas of the section due to the vehicle's speed.

The movements of dynamic closure surfaces affect the local behaviour of different joint contacts significantly. Therefore, it is necessary to take into account the shearing stress in the vicinity of the hinges and the compression stress in areas that are farther from them. In addition, the high friction coefficients of elastomers must be considered to achieve stable behaviour of the rubber profiles. (Friction parameters can be modified by applying different types of surface coatings.)

Design simulation of these components requires tools that provide accurate results for fast, but realistic, analyses. Design cycles are short and, therefore, not always compatible with the time required to physically test components, particularly during bidding processes. Virtual prototyping and qualification enable designers to run through more iterations, while considering complex interactions between loading regimes. This results in improved products. This is the case with rubber-based sealing products in both bidding of, and setting up, production lines. Virtual product design validation permits designers to reduce the number of prototypes and to benefit from considerable reductions in time and expense.

Virtual validation faces different problems, such as difficulties in dealing with rubber incompressibility from a numerical point of view (the finite element methods frequently break down when applied to engineering elastomers) or processing excessive deformation in rubber components, which usually leads to mesh entanglement, etc. Additional problems involve the initial rubber configuration that is necessary for installation on the door. Classical models use the curve centreline as a sweep model to deploy a characteristic section of rubber. Even when such an approach is possible, it causes disruptions because the bending of the rubber profile that is required when installing it on the door is neglected. These curvatures change the initial shape of the section and induce stresses in the rubber. Sometimes, installing the rubber in place produces undesired folds, which must be identified as early as possible (see Figure 2).

Figure 2 Example of an undesired type of fold to identify at an early stage of the design process (see online version for colours)

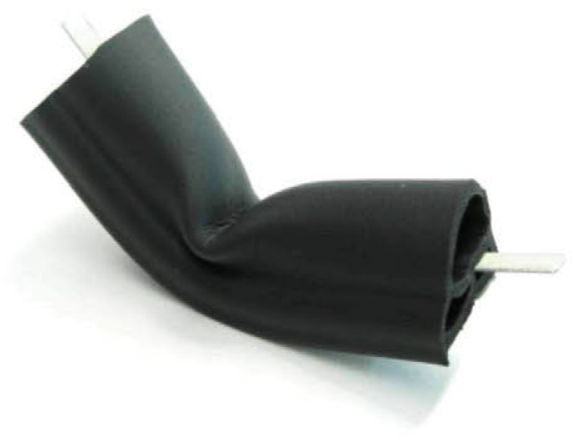


Our interest in this work involves more realistic approaches that make it possible to increase the reliability of predicted door-closing forces, taking into account rubber properties and geometric configurations, with specific attention given to the sensitivity of forces to rubber's mechanical parameters. This will improve the prediction capabilities of virtual design components and, as a direct consequence, enable more realistic virtual validations platforms to be produced.

The remainder of this paper is organised as follows: In Section 2, mathematical formulations for these problems are derived. In Section 3, a particular strategy for deriving the initial configuration and stresses for rubber's profile is provided. The required transformation to the runner's profile is addressed by means of the quaternion theory. Later, in Section 4, numerical approaches that are followed in virtual analyses are discussed. In Section 5, a sensitivity analysis and its results are discussed, including a comparison with the results of physical tests. Finally, Section 6 is devoted to the conclusions that can be drawn from the work and to the identification of questions or effects that remain unanswered and require considerable analysis.

\section{Problem formulation}

The classical theory of rubber-like materials was well addressed in Ward (1983). More recently, a discussion of different models of mechanical behaviour can be found in Muhr (2005) and Garcia Tarrago et al. (2009). In regards to the modelling of their properties for finite element analysis, broad reviews of mathematical models for the mechanical properties of rubber-like materials can be found in Charlton and Yang (1994). In addition, Mackerle $(1998,2004)$ gave a bibliographical review of the mathematical models and finite element simulations of rubber and rubber-like materials.

The neo-Hookean model assumes the strain energy density to be a linear function of $I_{1}$ and the Mooney-Rivlin model assumes the strain energy density to be a linear function of $\left(I_{1}, I_{2}\right)$. Comparisons of the Mooney-Rivlin model, Ogden model, foam model, neo-Hookean model, Yeoh model, Arruda-Boyce model and the Van der Waals model for rubber-like materials can be found in Chagnon et al. (2005). Each of these well-known models is able to closely match the experimental data for certain materials in a particular range of strain. The variety of complicated behaviours of rubber-like materials makes it very difficult to interpret them with a single model.

Consider an elastic body that initially occupies a region $\Omega_{X}$ with a boundary $\Gamma_{X}$. The deformation of a material particle $X \in \Omega_{X}$ at time $t$ is described by $X=\varphi(X, t)$, and the displacement of the particle $X$ is defined by

$$
u(X, t)=\varphi(X, t)-X=x(X, t)-X
$$

where $\varphi$ is the injective in $\Omega_{X}$ and the deformation is orientation-preserving. The reference frame is considered to be a rectangular Cartesian system. The deformation gradient $F$, Green-Lagrangian strain, $E$, and Green deformation tensor, $G$, are defined by:

$$
\mathrm{d} x=F \mathrm{~d} X ; \quad F_{i j}=\frac{\partial x_{i}}{\partial X_{j}}=\frac{\partial u_{i}}{\partial X_{j}}+\delta_{i j} .
$$


The distance between two material points before and after the deformation is given by:

$$
\mathrm{d} x^{T} \mathrm{~d} x=\mathrm{d} X^{T} F^{T} F \mathrm{~d} X
$$

where

$$
C=F^{T} F .
$$

(called the right Cauchy-Green deformation tensor). Since it is positive if $\mathrm{d} X \neq 0$, there is a principal orientation in which the tensor is diagonal:

$$
C=\left(\begin{array}{ccc}
\lambda_{1}^{2} & & \\
& \lambda_{2}^{2} & \\
& & \lambda_{3}^{2}
\end{array}\right) \text { and } F=\left(\begin{array}{lll}
\lambda_{1} & & \\
& \lambda_{2} & \\
& & \lambda_{3}
\end{array}\right)
$$

where $\lambda_{i}(i=1,2,3)$, called the principal stretch ratios. The change in distance of the material point in relation to points in its neighbourhood is:

$$
\mathrm{d} x^{T} \mathrm{~d} x-\mathrm{d} X^{T} \mathrm{~d} X=\mathrm{d} X^{T}\left(F^{T} F-I\right) \mathrm{d} X=2 \mathrm{~d} X^{T} E \mathrm{~d} X .
$$

Here the Green-Lagrangean strain tensor is defined as

$$
E=\frac{1}{2}\left(F^{T} F-I\right) ; \quad \varepsilon_{i j}=\frac{1}{2}\left(F_{k i} F_{k j}-\delta_{i j}\right) .
$$

The principal strains are related to the principal stretch ratios by:

$$
2 \varepsilon_{i i}=\lambda_{i}^{2}-1 .
$$

Three invariants of the right Cauchy-Green deformation tensor are defined as

$$
\begin{aligned}
& I_{1}=\lambda_{1}^{2}+\lambda_{2}^{2}+\lambda_{3}^{2} \\
& I_{2}=\lambda_{1}^{2} \lambda_{2}^{2}+\lambda_{1}^{2} \lambda_{3}^{2}+\lambda_{2}^{2} \lambda_{3}^{2} \\
& I_{3}=\lambda_{1}^{2} \lambda_{2}^{2} \lambda_{3}^{2}=(\operatorname{det}(F))^{2}=J^{2}
\end{aligned}
$$

where $J$ represents the volume deformation in the following way:

$$
\mathrm{d} v=J \mathrm{~d} V .
$$

For incompressible materials, there is a constraint of incompressibility, making it possible to rewrite the previous equations (1-12) in accordance with:

$$
J=\lambda_{1} \lambda_{2} \lambda_{3}=1 .
$$

Specific modelling for hyper-elasticity can be found in Lin (2009). Actually, this new model is a numerical interpolation algorithm for the construction of the strain energy density function that is obtained from its mechanical characteristics (see Figure 3). In that case, specific hints are provided to derive the tangent modulus tensor that is necessary to improve convergence in numerically solving equations. 
Figure 3 Strain-stress curves for rubber 1914090100 used as a main component for door profiles H603 under different loads
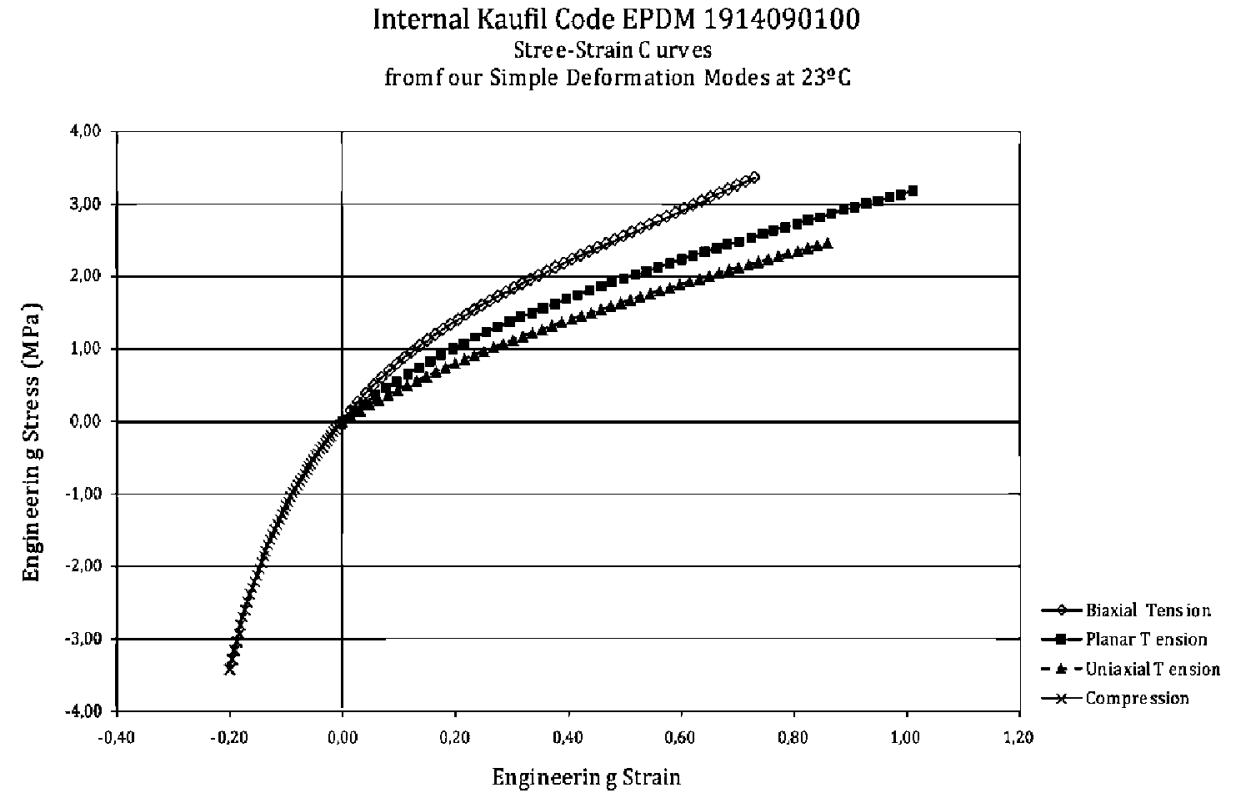

\section{Geometric modelling}

Geometric modelling is an important effect that is addressed in this work. It introduces relevant differences in outputs from simulation models, pending adoption of a hypothesis concerning the initial conditions of the rubber.

In the approach presented, the functional $\varphi$ introduced in equation (1) is a convolution of two additional functions. The first allows the transformation of its initial configuration to the in-place initial state and the second takes care of the non-linear displacements due to contacts and other actions involved in this problem. The laws that govern this second function were introduced in the previous section. Now it is time to describe the first function and its formulation. This is a non-linear, three-dimensional profile formulation. The deformed geometry of the profile is described by its deformed axis and the rotation of the cross-sections. Thus, the configuration space of the profile consists of:

- The linear space of the position vector of the deformed axis, and the non-linear space of rotations of cross-sections. The configuration space is thus a non-linear manifold.

- The non-linearity of spatial rotations requires special treatment, which makes the study of three-dimensional profiles interesting and challenging.

There are a number of ways to choose the parameters that describe spatial rotations. As three independent parameters suffice to describe locally the spatial rotation, three-parameter formulations have been used almost exclusively in beam theories. While the rotational vector possesses a clear geometrical meaning, which is advantageous, a disadvantage is that the discretisation of rotations using a standard additive interpolation spoils the objectivity of rotational strain measures outside the 
interpolation points. Several alternative interpolation strategies were discussed by Romero (2004), where the quaternions for rotational update and interpolation purposes are also presented.

A promising alternative for the parameterisation of rotations is the four-parameter rotational quaternion. The use of rotational quaternions in beam theories has been limited so far to the construction of numerically-stable and efficient, local, computational algorithms that avoid singularity problems and accelerate computations. However, the quaternions not only prove to be numerically stable and computationally efficient tools for algebraic operations with rotations, but also offer an alternative description of rotations in four dimensions.

This paper presents an efficient method to smoothly transform the straight rubber profile into a final shape that corresponds to the geometry of the vehicle's body. The method makes use of a moving frame that is adapted as proposed by Lazarus (1995). A simple propagation equation that involves the moving frame, which accounts for the transition between two consecutive points of a 3D polyline, is introduced. The curve transformation is accomplished using the interpolation of the quantities involved in this propagation equation. Depending on the type of interpolation, two algorithms are proposed to compute the intermediary shape of the curve. Moreover, a measure of the transformation is defined and minimised to distinguish a 'minimal transformation' that the user can modify by the addition of simple parameters.

A classical way to study the geometry of a curve is to define an adapted frame along the curve, whose members are either tangent to, or perpendicular to, the curve (Bishop, 1975; Faugeras, 1993). This approach is easier to implement than the spline-oriented one introduced by Weiss (2010).

As mentioned in the introduction, it is considered to be a parameterised curve $\Gamma$ that is defined by the interval $[0,1]$, sampled with the step $1 / n$. If $\Re$ is an adapted frame for $\Gamma$, it is possible to express $\Gamma((i+1) / n),(i=0,1, \ldots, n-1)$ in the local frame $(\Gamma(i / n), \Re(i / n))$ by the following progression equation (see Figure 4)

$$
\Gamma\left(\frac{i+1}{n}\right)=\Gamma\left(\frac{i}{n}\right)+\frac{1}{n} \mathfrak{R}\left(\frac{i}{n}\right) \gamma\left(\frac{i}{n}\right) .
$$

Figure 4 From $\Gamma(i / n)$ to $\Gamma((i+1) / n)$

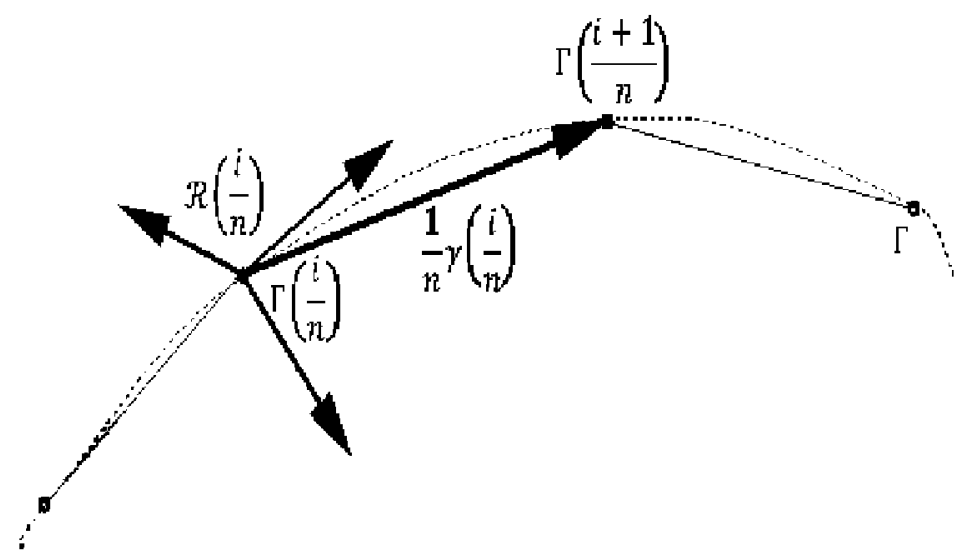


Now, given two curves $\Gamma_{0}$ and $\Gamma_{1}$ it is possible to construct a curve $\Gamma_{t}$ going from $\Gamma_{0}$ to $\Gamma_{1}$. To do so, the quantities $\left(\gamma_{t}(i / n), \Re_{t}(i / n)\right)$ from $\left(\gamma_{0}(i / n), \Re_{0}(i / n)\right)$ to $\left(\gamma_{1}(i / n), \Re_{1}(i / n)\right)$ are interpolated, and then the polyline $\Gamma_{t}(1 / n)$ is recovered at any time $t$ in $[0,1]$, using equation (14).

The relatively-parallel adapted frame, or RPAF, that is introduced is an alternative to the most commonly used Frenet Frame or FF, (Do Carmo, 1976), which is characterised by the nullity of the second component of the angular velocity expressed in the local frame.

Starting with an arbitrary frame, the frame is propagated along the curve by rotating it as little as possible, while keeping the frame adapted to the curve. As stated in Klok (1986), it has some advantages over the FF. Apart from its minimal rotation, it is defined at every point for every regular curve, twice continuously differentiable. In particular, the RPAF is defined where the curvature vanishes, although this is not the case for the FF.

From Bishop (1975), it may be seen that an RPAF is characterised by the nullity of the first component of the angular velocity expressed in the local frame. More precisely, its angular velocity takes the form of $\Omega=v \kappa b$, where $v, \kappa$ and $b$ are defined as for the FF: if $(t, f, g)$ are the axis of the frame $\Re$, the angular velocity $\Omega$ must be of the form $-\lambda t+\mu g$. Identifying $t^{\prime}$ and $\Omega \times t$ we see that

$$
v \kappa n=\mu f .
$$

Thus,

$$
f= \pm n ; \quad g= \pm b ; \quad \mu= \pm v \kappa ; \quad \lambda= \pm v \tau
$$

where $n, b, v, k$ and $\tau$ are, respectively, the normal, the binormal, the velocity, the curvature and the torsion of the curve $\Gamma$. Hence, in regard to the velocity, the components of $\Omega$ are equal to the curvature and the torsion of the curve.

Now after the interpolation of $\Omega_{t}$ from $\Omega_{0}$ to $\Omega_{1}$, keeping the first component equal to zero, it will be possible to derive a frame $\Re_{t}$ that is an RPAF at each time. Moreover, the curvature is given by the norm of the angular velocity $\Omega_{t}$, up to the velocity $v_{t}=(1-t) v_{0}+t v_{1}$.

For a polyline, a good approximation of the RAPF can be constructed. For this, first note that

$$
\lim _{u \rightarrow 0} \frac{\cos ^{-1}\langle t(s), t(s+u)\rangle}{u}=v(s) \kappa(s) .
$$

Hence, if $\alpha_{i}$ is the angle formed by $t(i / n)$ and $t((i+1) / n)$, it is seen that $n \alpha_{i}$ converges uniformly to $v(s) \kappa(s)$.

In this way, the following recursive equation is obtained:

$$
\Re_{i+1}=\Re_{i} \exp \left(\alpha_{i} b_{i}\right)
$$

so that $\Re_{i+1}$ is obtained by rotating $\Re_{i}$ about $b_{i}$ an angle $\alpha_{i}$, as stated in Klok (1986).

The linear interpolation of the angular velocity linked with the relatively parallel adapted frame can be expressed by

$$
\Omega_{t}=(1-t) \Omega_{0}+t \Omega_{1}=(1-t) v_{0} \kappa_{0} b_{0}+t v_{1} \kappa_{1} b_{1}
$$


and by the triangular inequality

$$
\left|\kappa_{t}\right| \leq\left|\kappa_{0}\right|+\frac{1}{\left(1+\frac{v_{0}}{v_{1}}\right)-\frac{1}{t} \frac{v_{0}}{v_{1}}}\left(\left|\kappa_{1}\right|-\left|\kappa_{0}\right|\right)
$$

which shows that $\left|\kappa_{t}\right|$ is included between $\left|\kappa_{0}\right|$ and $\left|\kappa_{1}\right|$. Actually, it is possible to obtain the same interpolation for the curvature and the torsion as for the Frenet frame. Indeed, in the local frame of a RPAF, the binormal is expressed by $\left(0,-\sin \theta_{i}, \cos \theta_{i}\right)$ (see Bishop, 1975 ), where $\theta$ is a primitive of the torsion (see Figure 5 ) Thus,

$$
\Omega_{i}=n \alpha_{i}\left(0,-\sin \theta_{i}, \cos \theta_{i}\right) .
$$

Figure 5 The angles $\alpha_{i}$ and $\theta_{i}$

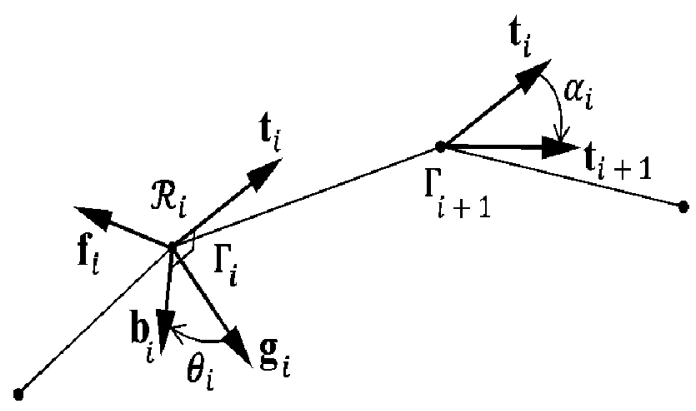

A linear interpolation of $v \kappa$ and $v \tau$ can be derived by replacing the linear interpolation of $\Omega_{i}$ by the linear interpolation of the angles $\alpha_{i}$ and $\theta_{i}$.

To give more flexibility to this interpolation, the user can add a scalar field along the curve. This field represents an extra twist of the associated frame. The user assigns a real value to some points of the curve. Those values are then interpolated along the curve and interpreted as angles. Each frame is then rotated about the tangent using these angles. Hence, the associated frame $\Re$ (that is, the FF or the RPAF) is replaced by

$$
\bar{\Re}=\Re r_{\varphi}
$$

where $r_{\varphi}$ is a rotation of angle $\varphi$ about the $x$-axis.

Using a constant twist of angle $\pi$, it is possible to straighten any curve of constant length. As a matter of fact, it is possible to transform a curve into one that has a twisted angular velocity, using quaternion slerping (spherical linear interpolation). As this corresponds to a linear interpolation of the angular velocity, at a half-way point, we will have $\Omega_{1 / 2, i}=0$. From this and the fact that the length is linearly interpolated, at time $t=1 / 2$ the interpolated curve is a straight segment of the same length as the initial curve. Note that the angular velocity follows a geodesic (i.e., a segment) that passes through zero. Hence, the transformed path of rotation is also a geodesic in the rotational space. More precisely, as the curve is straightened, the associated moving frame is retracted over the identity following the geodesics. 
In the implementation presented, we have used the RPAF instead of the FF, so that we have two modes of interpolation, apart from the extra parameter linked to the frame twist. One mode uses quaternion slerping to correspond to a linear interpolation of the angular velocity and the other mode uses interpolation of angles. Below, the algorithm steps are summarised for both methods, calling the two methods Q and A (for quaternion and angle) respectively.

\section{Preprocessing of $Q$}

1 Evaluate tangents $t_{i}$ at points $\Gamma_{i}$

2 Compute the angles $\alpha_{i}=\angle\left(t_{i}, t_{i+1}\right)$, the binormals $b_{1}=\left(t_{i} \times t_{i+1}\right) /\left\|t_{i} \times t_{i+1}\right\|$, and the quaternions $q_{i}=\left(\cos \left(\alpha_{i} / 2\right), \sin \left(\alpha_{i} / 2\right) b_{1}\right)$

3 Propagate the frame $\Re_{0}=\left(t_{0}, f_{0}, g_{0}\right)=\left(t_{0}, n_{0}, b_{0}\right)$ using equation (2)

4 Calculate the local coordinates $\gamma_{i+1}=n \Re_{i}^{-1}\left(\Gamma_{i+1}-\Gamma_{i}\right)$.

Note that, in step 3 of this implementation, a conversion for rotations into quaternion allows the calculation of propagation using equation (2).

Interpolation at time $t$ with $Q$

1 Calculate $\left(\Gamma_{t, 0}, \Re_{t, 0}\right)$ from $\left(\Gamma_{0,0}, \Re_{0,0}\right)$ and $\left(\Gamma_{1,0}, \Re_{1,0}\right)$ and $t$

2 Compute for each $i: q_{t, i}=\operatorname{slerp}\left(q_{0, i}, q_{1, i}, t\right)$ and $\gamma_{t, i}=\operatorname{lerp}\left(\gamma_{0, i}, \gamma_{1, i}, t\right)$

3 Recover $\Gamma_{t, i}$.

Note that step 1 only influences the relative position of the interpolated curve and not its shape. More precisely, the geometry of the transformation is independent of the relative positions of the curves, considering the involved entities in local coordinates.

In this implementation, lerping (linear interpolation) and slerping for the point $\Gamma_{t, 0}$ and the frame $\Re_{t, 0}$ were used separately.

Preprocessing of $A$

This is the same as for $Q$, but it is necessary to add the calculation of $\theta_{i}=\angle\left(g_{i}, b_{i}\right)$ in step 4 .

Interpolation at time $t$ with $A$

1 This is the same as for $Q$

2 Compute for each $i: x_{t, i}=\operatorname{lerp}\left(x_{0, i}, x_{1, i}, t\right)$, with $x=\alpha, \theta$ or $x=\gamma$

3 Recover $\Gamma_{t, i}$.

In summary, a new technique to fold smoothly or straighten a polyline, using adapted moving frames, has been presented. The relationship between the two algorithms that have been designed for the polylines and the underlying continuous parameterisations has also been shown. This approach is particularly adapted to the control of local geometry (curvature and torsion), as well as global geometry (length). 


\section{Numerical approach}

In accordance with the previous sections, a linear configuration of rubber is adopted. A deformation path that was modelled by quaternions was followed by using a large deformation approach, as well as an incompressible hyper-elastic constitutive law. It is interesting to note that this procedure is carried out by morphing the initial mesh along the deformation path by small steps to avoid topological distortions that could make the transformed mesh unusable for FEM simulations (see Figure 6).

Figure 6 Incremental transformation to permit the estimation of initial stresses (see online version for colours)

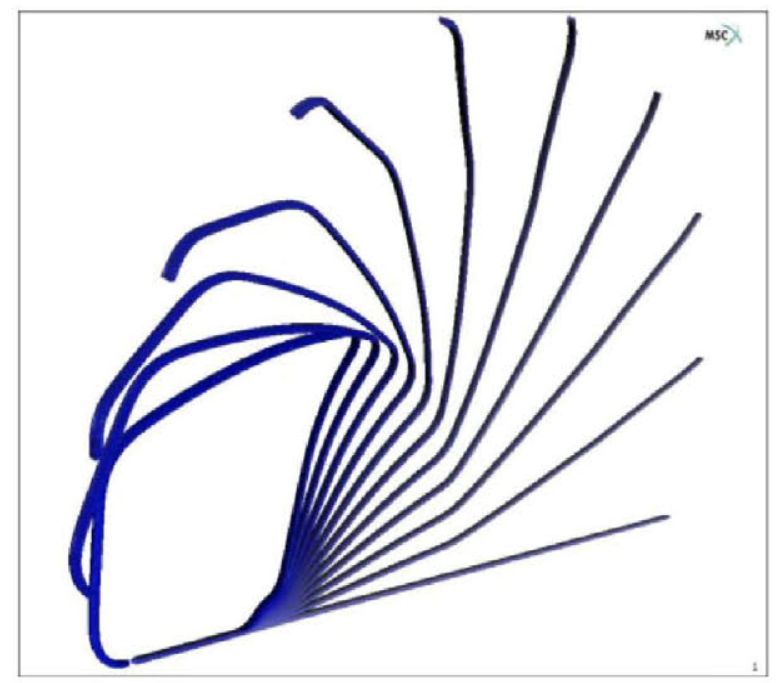

This produced the initial rubber configuration that was tested numerically to estimate the closing force and sealing pressures.

Mechanical parameters for the rubber, according to the problem formulation section, were tested for the Ogden model with two terms and the Third Order Deformation with the following parameters:

\begin{tabular}{lccc}
\hline MODEL & & & \\
\hline Ogden & Moduli & Exponents & \\
\hline & 2.6106 & 0.3717 & \\
Third order deformation & 0.00045393 & 5.56565 & \\
& & & \\
& $\mathrm{C}_{10}=0.0555223$ & $\mathrm{C}_{01}=0.0644285$ & $\mathrm{C}_{11}=0$ \\
& $\mathrm{C}_{20}=0$ & $\mathrm{C}_{30}=0.0004001$ & \\
\hline
\end{tabular}

Coherence data between real and simulated tests are presented in Figure 7. 
Figure 7 Real vs. simulated deformations for different material models

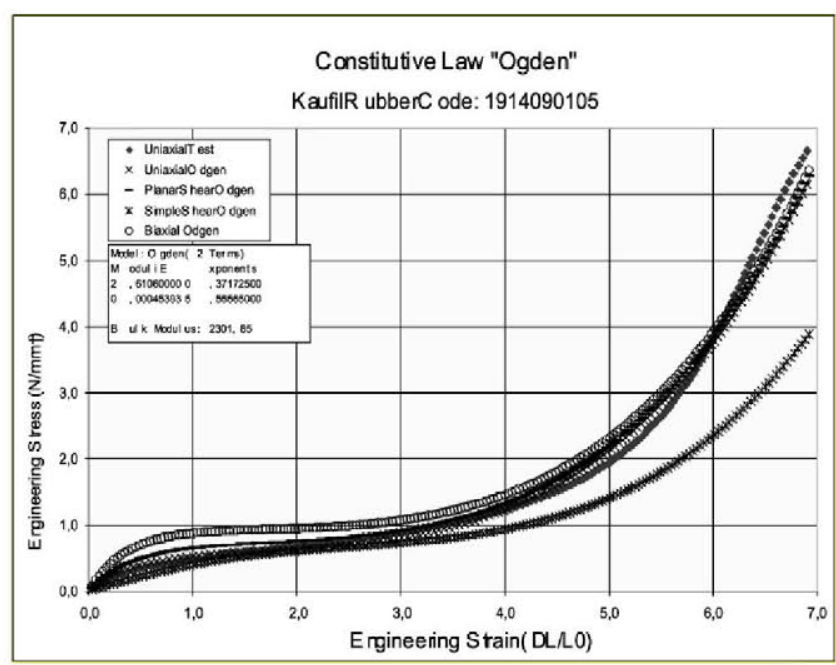

(a)

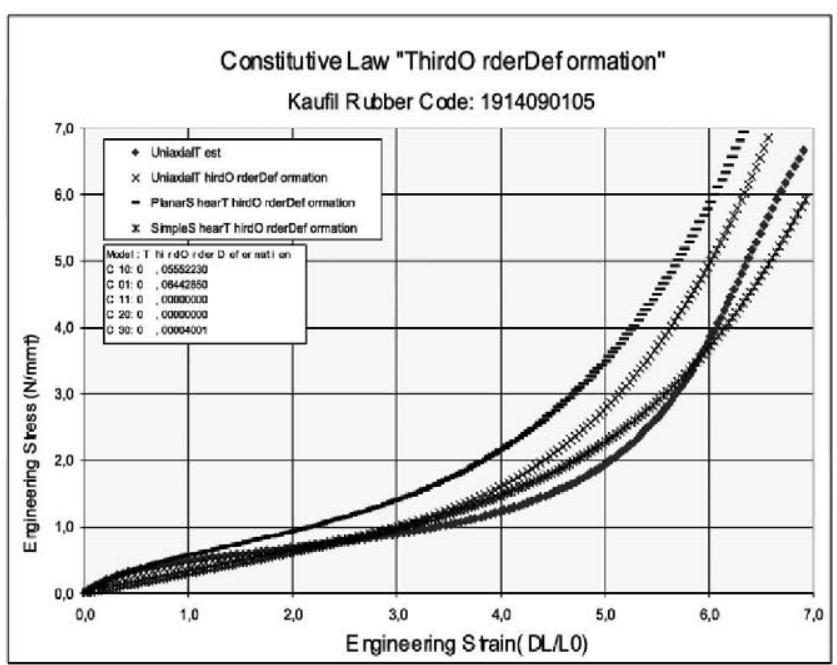

(b)

In Figure 8, the initial rubber configuration shows the setting-up stress level in particular sections of the rubber (the colours show the equivalent Cauchy stresses produced by the rubber installation). More details are provided in Figure 9, where the impact on sections is shown when deformation occurs. The deformed rubber and deformed transversal section, which are colour-coded for equivalent Cauchy stress levels, show the stick-slip phenomenon with its left side lip. A friction model was considered to simulate reality as closely as possible. Friction involves the characteristics of the surface, such as surface roughness, temperature, normal stress, and relative velocity. For a given normal stress or normal force, the friction stress or force behaves like a step function based upon the value of the relative sliding velocity $v_{r}$ or the tangential relative incremental displacement $\Delta \boldsymbol{u}_{t}$. This is the stick-slip model (Mijar and Arora, 2004). Since this discontinuity in the 
friction value may cause numerical difficulties, different approximations of the step function have been used (Strömberg and Klarbring 2010) and a Coulomb-oriented model with bilinear numerical approach was adopted in this work to avoid these difficulties. The bilinear model assumes that the stick and slip conditions correspond to reversible (elastic) and permanent (plastic) relative displacements, respectively. The clear resemblance to the theory of elasto-plasticity will be used to derive the governing equations.

Figure 8 Initial rubber on site configuration with no pressure from the door (see online version for colours)

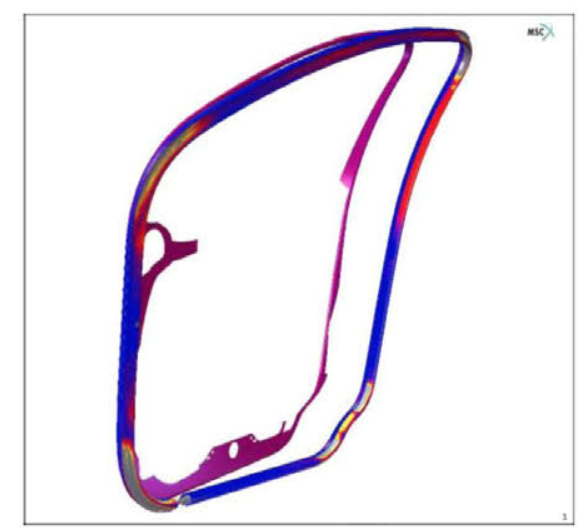

Figure 9 Left: undeformed rubber with nominal section. Right: deformed rubber with transversal section showing initial and deformed shapes (see online version for colours)

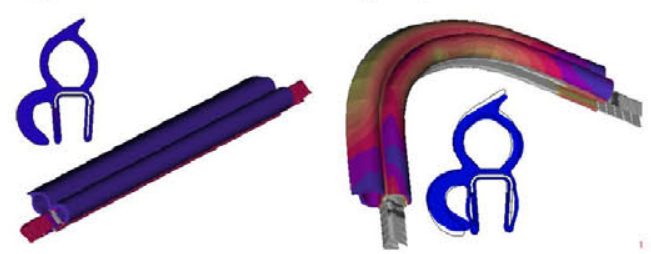

Although the MSC.Marc $\mathcal{C}$ non-linear finite element solver was used to model the case study, the choice of the solver type does not limit the generality of the methodology developed, as the key factor is the initial condition for starting the simulation and its initial stress status. In fact, the implementation of this geometric morphing is carried on outside of the solver itself (Marc in this particular case) and performed by a homemade script in VBasic ${ }^{\circledR}$, allowing it to configure those transformations for specific nodes and along different increments. The final geometry is rewritten as if it was obtained from MSC.Mentat $\mathbb{C}$. The type of finite elements selected is like a three-dimensional brick that can be formed as required. These finite elements are formulated according to Herrmann's Formula (eight-node iso-parametric with an additional ninth node for pressure). Specific hyper-elastic models have been considered as constraints and particular constitutive laws, in accordance with measured material characterisation parameters that have already been presented in this section.

Because of rubber's flexibility, it is important to remember the highly nonlinear relationship that it exhibits. Due to the contact problem, it is not easy to identify the contact location. Also, the cost of the Lagrange multiplication method is high 
due to extra variables for contact modelling (both deformable-to-rigid and deformable-to-deformable). In addition, it is necessary to consider friction due to surface imperfections, stick-slip motion, or material softening because of heat in the contact area, etc. (see Figure 9).

The boundary conditions come from moving the axis to adapt it to its mounting position. Then, according to the geometric transformations presented above, the different meshing sections are defined and contact constraints are defined against a door structure modelled with rigid surfaces. Here, an iterative solution process using the constitutive model presented in the problem formulation section with friction activated, is begun. It is important to remember that three different materials, with different mechanical properties, are involved:

- steel for the structural section

- the rubber basic component, modelled with a hardness of Shore $70 \mathrm{~A}$

- a special coextruded soft rubber mixture that was modelled as foam and provides adequate properties for contacts.

Lack of convergence can take place due to input errors, improper modelling of a physical phenomenon, or, sometimes, real physical instabilities. A good strategy to reduce a numerical lack of convergence is to use an automatic remeshing option, taking into account the gradient of strain as an indicator of the entanglement of elements (Ha et al., 2010). This strategy makes it possible to simulate specific nonconformities like the one shown in Figure 2, as can be verified in Figure 10.

Figure 10 Deformed rubber with activated measures to increase convergence rating (see online version for colours)

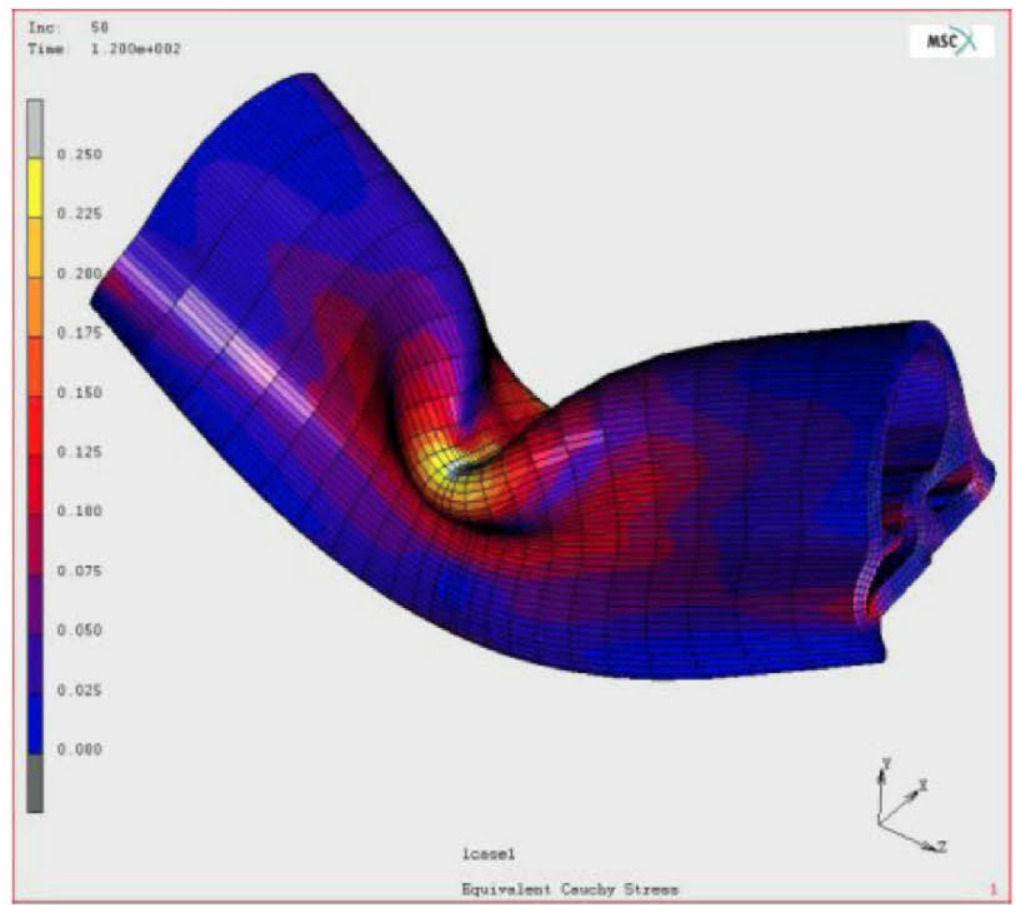


Figure 11 shows different hypotheses that have been analysed to evaluate the influence of the initial configuration on the door-closing force. Various cases were analysed to identify operating factors like:

a required door-closing force

b sealing pressure after the door has been closed.

Additional analyses were performed under the umbrella of a sensitivity test by changing different parameters and looking for the impact on functional performance, like those that were mentioned previously. These are the types of capabilities that are presented by the virtual design and evaluation tool, which is discussed in this paper.

Figure 11 Different initial hypotheses. Left: classical, stress-free configuration (case ' $c$ ') Right: initial stresses due to setup are calculated, considered and displayed (case 'a') (see online version for colours)

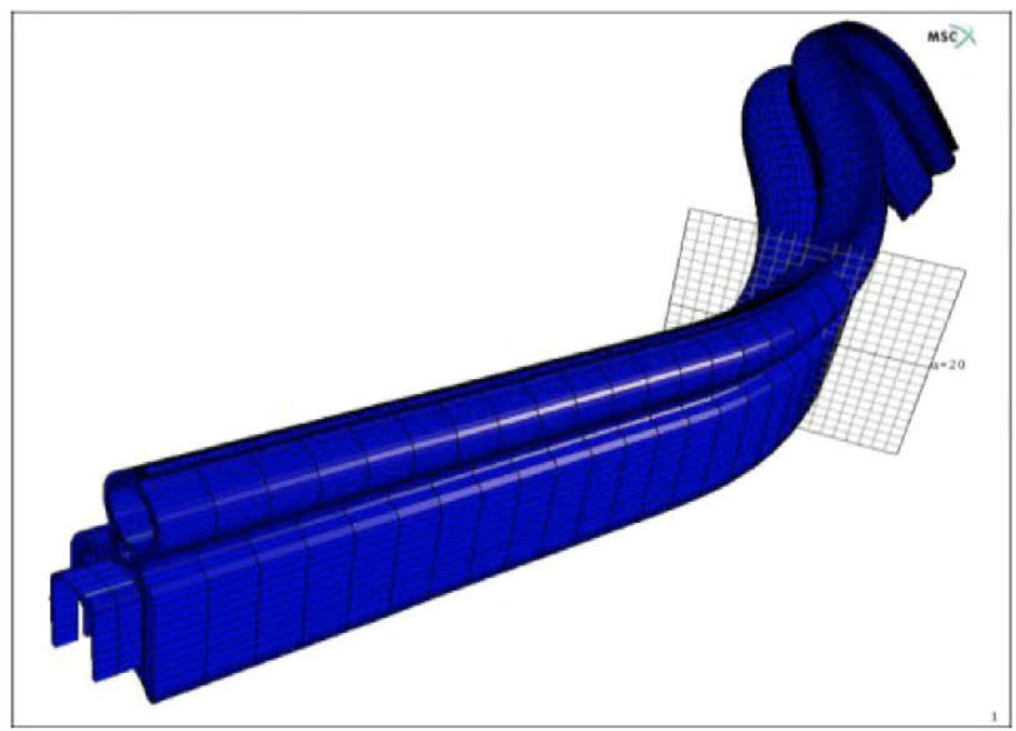

\section{Results}

In regard to specific simulation results, it is possible to note that the rubber close to the door articulations is excited mainly by shear stress. On the other hand, the main effects of sections far from these points are related to compression, as expected.

Three cases were analysed to evaluate stress levels:

a bending of the rubber profile with consideration to the initial stresses (see Figure 12)

b bending of the rubber profile without initial stresses (see Figure 13)

c extrusion of the section along the curved main line for rubber - obviously without stresses (see Figure 14). 
Figure 12 Left: a normal section in the middle of the curved profile with initial stresses considered (case ' $a$ '). Right: the same section after the door has been closed. The door is shown to identify the contact (see online version for colours)
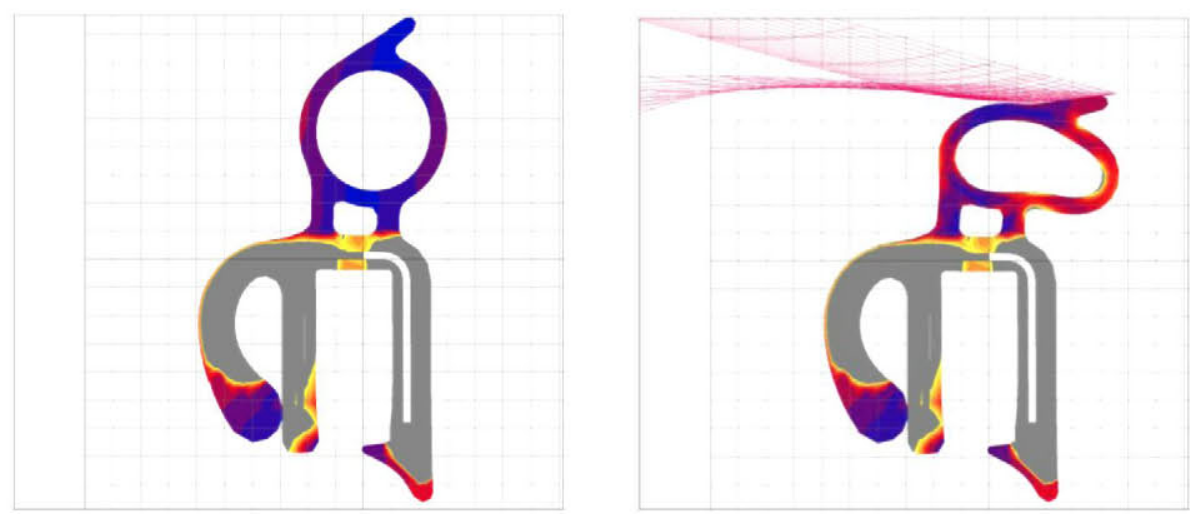

Figure 13 Left: a normal section in the middle of the curved profile without initial stresses considered (case ' $b$ '). Right: the same section after the door has been closed. The door is shown to identify the contact (see online version for colours)
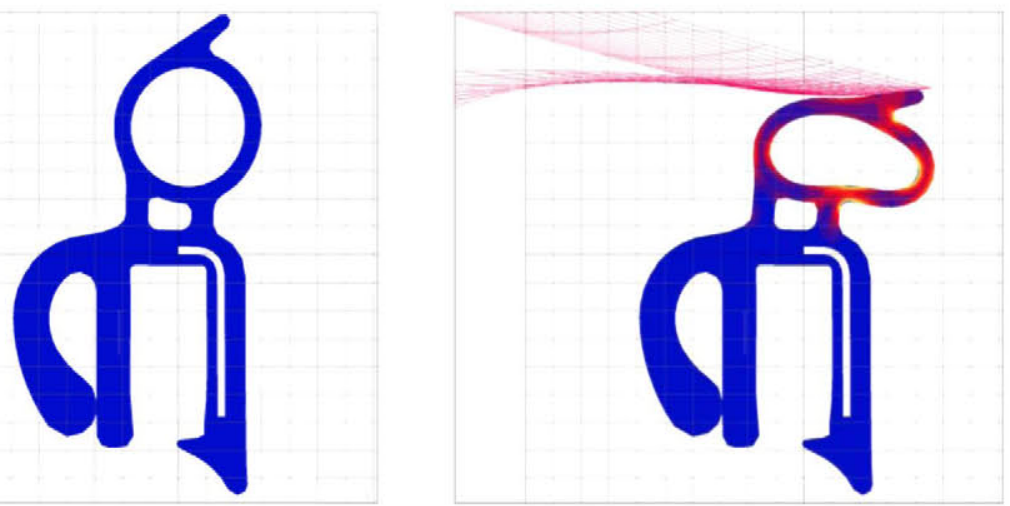

Figure 14 Left: a normal section extruded over the curved profile without initial stresses considered (case ' $c$ '). Right: the same section after closing the door (see online version for colours)
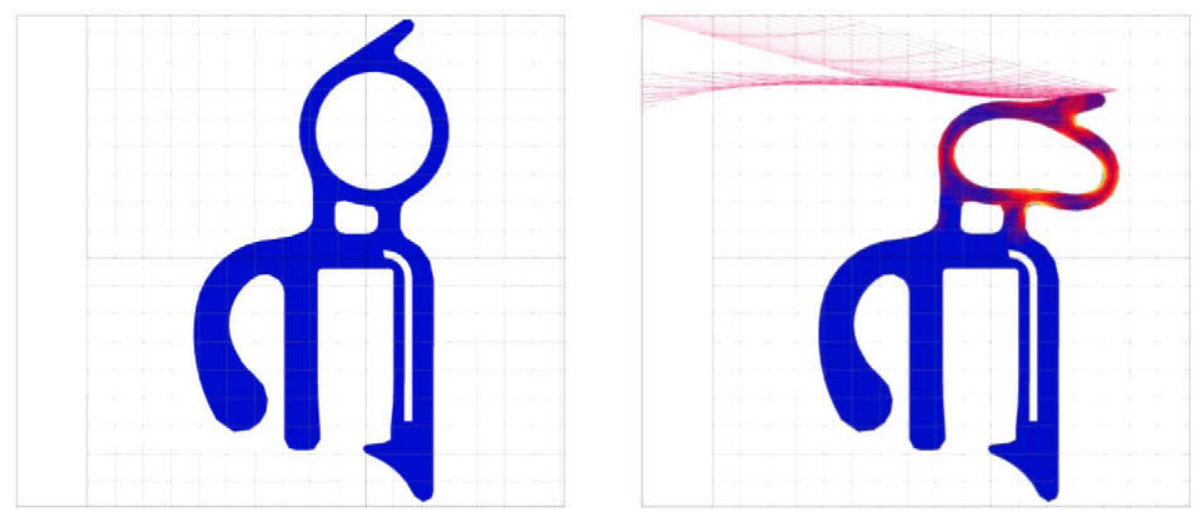
In all the cases, the rubber was morphed to its final configuration described by the theoretical design line and specific torsions where depicted, according to the expected final position of the rubber at those points. All these calculations were performed by specific homemade software tools implementing algorithms described in Section 3.

As shown in the right part of Figures 12-14, higher levels of final stresses are found in case (a) than in case (b) and case (c). The intensity of stress is reduced globally by $10 \%$ in each subsequent case.

The main justification for bending simulation processes is to determine how the rubber profiles adapt to the areas of vehicles that are designed to accommodate different anchor types (metal strip, clips, belt adhesive, etc.). Thus, the most critical defect in this process is the accumulation of material and stress, which promotes the formation of wrinkles on the joints and, thereby, breaking of the seal (see Figure 9). Hence, it is necessary to analyse the behaviour of tension and strain profiles along the anchor line to ensure that different sections fulfil their functionalities.

We can see from the examples in Figures 12-14 that the FEM extrusion geometries (case ' $c$ ') do not represent the real behaviour of the profile. Therefore, to avoid design errors arising from these geometries, it is important to work on the simulation process of bending (cases ' $a$ ' and ' $b$ ').

Subsequent to the forming or bending operations, it is essential to study the problem of contact in the door-closing simulation. Consideration should be given to:

- Studying the stability of the profile section in different positions of the contact to ensure a seal

- Verifying the continuity of contact and the closure pressure developed to meet the functionality (see Figure 15). In this sense, it is necessary to mention the importance of the design and development processes of the closing force. These forces must be balanced to seal the door closing forces of the vehicle examined under the prism of static and dynamic clamping forces (Ordieres-Meré et al., 2008).

Figure 15 Study of the contact surface along the rubber profile to determine the sealing pressure in a door closure (see online version for colours)

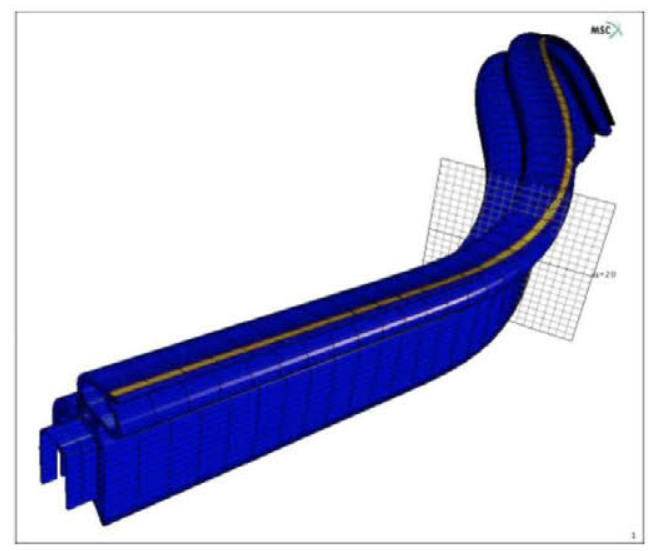

Looking at the door-closing forces in the three variants of numerical simulations that have been analysed in this work (see Figure 16 where the force is represented against time and the displacement at fixed sections), it is easy to realise that the sealing pressure 
is sensitive to each of the cases studied, where the final forces vary between $10 \%$ and the $25 \%$ in the analysed quasi-static case (i.e., avoiding additional dynamic forces arising from closing door speed and its mechanical acceleration).

Figure 16 Comparative analysis of required closing force considering different initial hypotheses

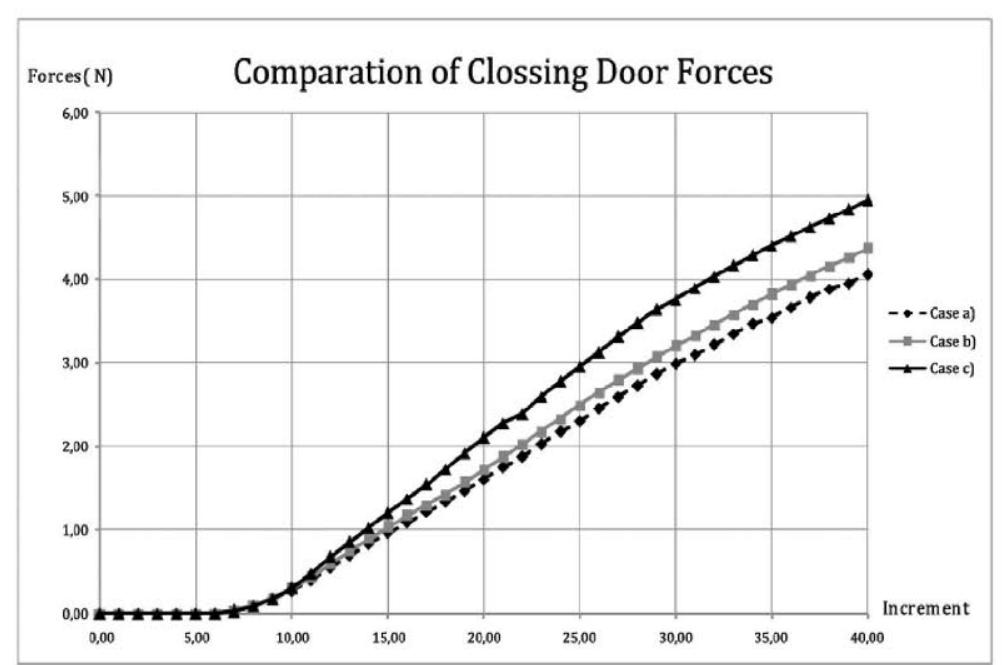

As the main goal is to compare different rubber sections for specific geometries, it is relevant to assess predictions from the simulation system against physical rubber (see Figure 17). To do this, a special test was defined, to calibrate the rubber mechanical parameters adopted for the constitutive law. The objective is not to validate the system as a whole, because many factors need to be controlled, like installation procedures, etc. Instead, it is necessary to validate the coherence at low levels and avoid dynamic effects like instabilities. It is important to remember that some rubber material models are unstable, in that an unstable material response may be obtained for certain strain levels. Then, if in the simulation of a configuration, strain levels corresponding to instability are reached, the Newton-Raphson nonlinear iterative solution may have difficulty in converging, and the calculated response may be physically unrealistic.

Figure 17 A detailed view of a tested section. Real configuration (Left). Numerical simulation for the test allowing a comparison of results for calibration (Right) (see online version for colours)
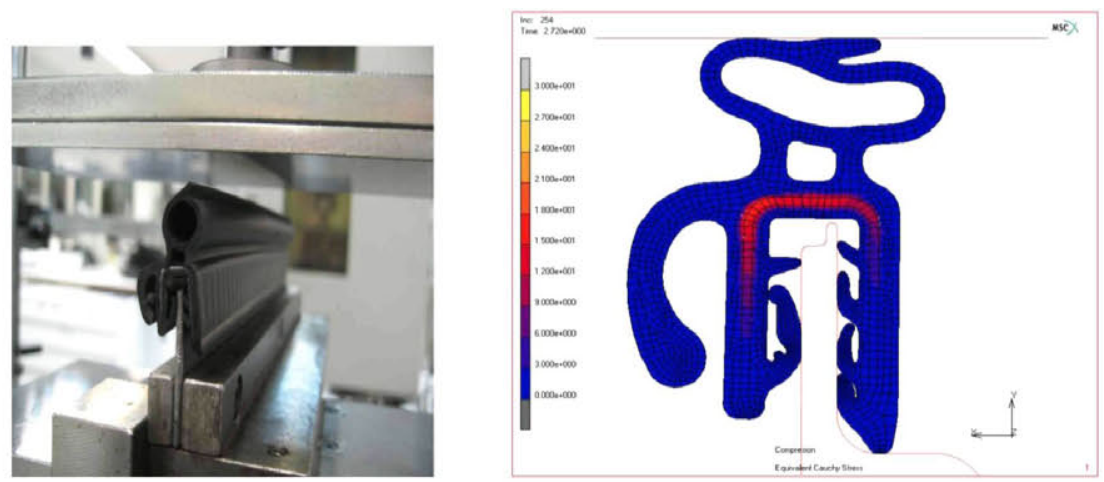
It permitted the measurement of the quasi-static forces involved by closing the door, per unit length of sealing solution. The section used in the numerical simulation, as well as a comparison of results, appears in Figure 18.

Figure 18 Analysis of closing forces per $200 \mathrm{~mm}$ of length of sealing rubber involved in the real test, as well as in the numerical simulation (see online version for colours)

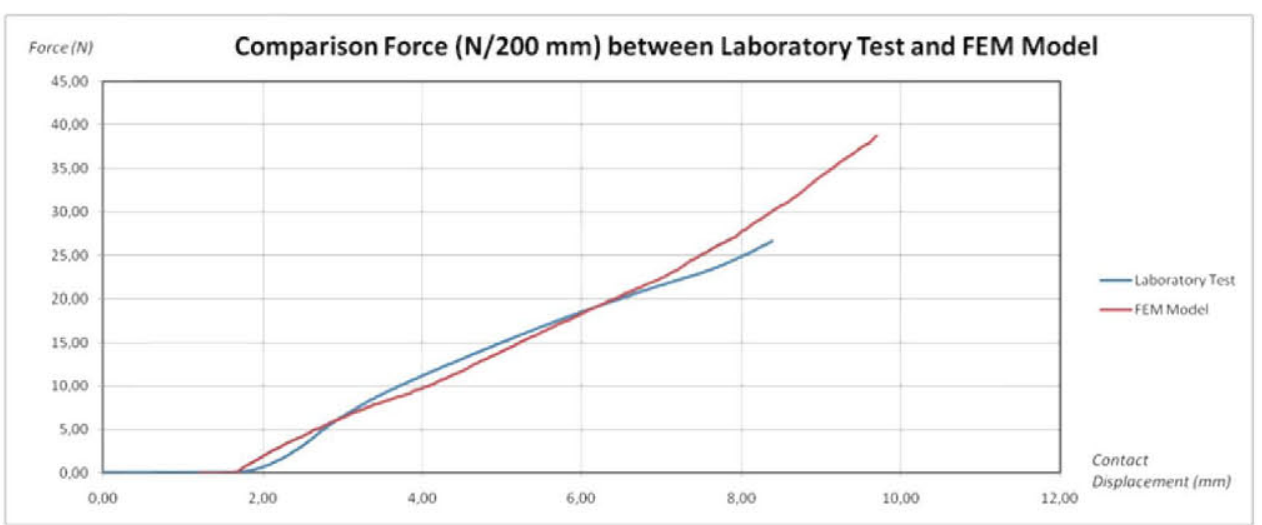

In the comparison of results, the coherence along the time dimension and the value of the forces indicate the quality of the tool.

Thus, it is possible to observe how the baseline scenario for bending significantly affects the results of door closing simulations. The process of bending in case ' $a$ ' provides more information about the deformation of the profiles, allowing design engineers to evaluate the stress state linked to the closing door effect to understand the real behaviour of sealing systems and optimise their design.

Also, these simulations allow designers to identify underperformance and suggest additions to sections, improving their capabilities to absorb geometric tolerances. As these simulations show where the sealing chain is broken or where wrinkles arise, it is possible to redesign the profile. These situations are quite close to the reactions to the supporting columns or where the rubber's shape changes quickly.

The designers' next step is to produce sensitivity simulations, modifying these geometrical parameters and attempting to identify their influence on performance criteria, like those mentioned in the previous section.

This sensitivity reveals a clear dependence of performance on the geometric designs of the profiles, where industry sealing trends are focused on variable extrusion systems to adapt to the increasingly complex designs and specifications of new vehicles.

\section{Discussion}

This paper presents a structured virtual design tool based on the finite element method, including modern constitutive laws and incompressibility constraints. This virtual tool includes integral strategies that enable it to become useful without undue delay, to 
simulate the steps in installing rubber and to predict the final closing forces more realistically and even estimate sealing overpressure as an additional guarantee of noise insulation or reduction.

This study reveals the relationship between the hypothesis concerning the initial configuration of the rubber and the estimated final value for the closing door. This factor has usually been neglected, but becomes relevant following this study in correctly assessing customers' requirements. It can be considered as a way to improve the design method of sealing sections and a convenient step for its optimisation. This type of tool is crucial for companies today, as it enables them to reduce design costs, as well as to be competitive by launching production processes that are oriented to prototype cycle reduction. It is even possible to use the tool in two different ways during bidding time first, for more accuracy in estimating material costs per metre of rubber and, second, for a higher degree of definition in designs that are offered to the customer, enabling a more robust solution to be produced.

In addition, if any discussion or modification is required, the impact on materials or functional parameters can be quickly analysed.

Finally, this type of tool enhances the added-value chain with the car manufacturer in such a way that the links between the car manufacturer and the rubber provider become stronger.

The step following this research will be to explore the capabilities for automatic optimisation by combining evolutionary strategies (part of the genetic algorithms family of tools) to create a new engine to drive sensitivity analyses. This will provide a more time-efficient search procedure that is compatible with a reduction of the mass of rubber used or, at least, a consideration of the latter.

\section{Acknowledgements}

This study was conducted in collaboration with Kaufil Sealing Technologies SA. The support of Mr. García-Forcada of that company is gratefully acknowledged. Part of the funds that supported this work were provided by the 'Ministerio de Educación' through research grant 'Salvador de Madariaga' (code PR2010-0014).

\section{References}

Bishop, R.L. (1975) 'There is more than one way to frame a curve', American Mathematical Monthly, Vol. 82, pp.246-251.

Chagnon, G., Marckmann, G. and Verron, E. (2005) 'A comparison of the Hart-Smith model with Arruda-Boyce and gent formulations for rubber elasticity', Rubber Chem. Technol., Vol. 77, pp.4724-4735.

Charlton, D.J. and Yang, J. (1994) 'A review of methods to characterize rubber elastic behavior for use of finite element analysis', Rubber Chem. Technol., Vol. 67, pp.481-507.

Do Carmo, M.P. (1976) Differential Geometry of Curves and Surfaces, Prentice-Hall, New Jersey.

Faugeras, O. (1993) 'Cartan's moving frame method and its application to the geometry and evolution of curves in the euclidean affine and projective planes', in Mundy, J.L., Zisserman, A. and Forsyth, D. (Eds.): Applications of Invariance in Computer Vision, No. 825, Springer-Verlag, pp.11-46. 
Garcia Tarrago, M.J., Gil-Negrete, N. and Vinolas, J. (2009) 'Viscoelastic models for rubber mounts: influence on the dynamic behaviour of an elastomeric isolated system', Int. J. of Vehicle Design, Vol. 49, No. 4, pp.303-317.

Ha, S-H., Choi, K.K. and Cho, S. (2010) 'Numerical method for shape optimization using T-spline based isogeometric method', Structural and Multidisciplinary Optimization, Vol. 42, No. 3 , pp. $417-428$.

Karen, I., Kaya, N., Ozturk, F. Korkmaz, I. (2010) 'Optimisation of vehicle engine mount system using simulation-based design approach', Int. J. of Vehicle Design, Vol. 52, Nos. 1-4, pp.38-53.

Klok, F. (1986) 'Two moving coordinate frames for sweeping along a 3D trajectory', Computer Aided Geometric Design, Vol. 3, pp.217-229.

Lazarus, F. (1995) A Geometrically Based Approach to 3D Skeleton Curve Blending, Rapport de Recherche No. 2458, INRIA

Lin, B. (2009) A New Model for Hyperelasticity, Acta Mech 208, pp.39-53.

Mackerle, J. (1998) 'Rubber and rubber-like materials, finite-element analyses and simulations: a bibliography (1976-1997)', Model. Simul. Mater. Sci. Eng., Vol. 6, pp.171-198.

Mackerle, J. (2004) 'Rubber and rubber-like materials, finite-element analyses and simulation, an addendum: a bibliography (1997-2003)', Model. Simul. Mater. Sci. Eng., Vol. 12, pp.1031-1053.

Mijar, A.R. and Arora, J.S. (2004) 'An augmented Lagrangian optimization method for contact analysis problems, 1: formulation and algorithm', Structural and Multidisciplinary Optimization, Vol. 28, Nos. 2-3, pp.99-112.

Muhr, A.H. (2005) 'Modeling the stress-strain behavior of rubber', Rubber Chem. Technol., Vol. 78, pp.391-425.

Nash, D.A., Lu, Y.C. and Anderson, M.E. (2009) 'Finite element modelling of elastomeric sealing components for cylinder-head cover noise and vibration prediction', Int. J. of Vehicle Design, Vol. 49, No. 4, pp.287-302.

Ordieres-Meré, J., Lopez, L.M., Bello, A. and Garcia, A. (2003) 'Intelligent methods helping the design of a manufacturing system for die extrusion rubbers', International Journal of Computer Integrated Manufacturing, Vol. 16, No. 3, pp.173-180.

Ordieres-Meré, J., Bello-García, A., Muñoz-Munilla, V. and Del-Coz-Díaz, J.J. (2008) 'Finite element analysis of the hyper-elastic contact problem in automotive door sealing', Journal of Non-Crystalline Solids, Vol. 354, Nos. 47-51, 1 December, pp.5331-5333.

Pan, X-Y. and Chaii, G-Z. (2009) 'Modelling of rubber mounts and applications for time response analysis of dynamic systems including elastomerics', Int. J. of Vehicle Design, Vol. 49, No. 4, pp.259-274

Piateck, R. (1987) 'Operation, safety and comfort', in Hucho, W-H. (Ed.): Aerodynamics of Road Vehicles, Butterworth \& Co. Ltd., London, ch. 6, pp.236-259.

Romero, I. (2004) 'The interpolation of rotations and its application to finite element models of geometrically exact rods', Comput. Mech., Vol. 34, pp.121-133

Stenti, A., Moens, D., Sas, P. and Desmet, W. (2006) 'Development of a numerical modelling methodology for the NVH behaviour of elastomeric line connections', International Conference on Noise and Vibration Engineering, Belgium.

Strömberg, W. and Klarbring, A. (2010) 'Topology optimization of structures in unilateral contact', Structural and Multidisciplinary Optimization, Vol. 41, No. 1, pp.57-64.

Wagner, D.A., Morman, Jr., K.N., Gur, Y. and Koka, M.R. (1997) 'Nonlinear analysis of automotive door weatherstrip seals', Finite Elements in Analysis and Design, Vol. 28, No. 1, 1 December, pp.33-50. 
Ward, I.M. (1983) Mechanical Properties of Solid Polymers, Wiley, New York.

Weiss, D. (2010) 'Feature-based spline optimization in CAD: a step towards geometrybased structure creation', Structural and Multidisciplinary Optimization, Vol. 42, No. 4, pp.619-631. 